\title{
Screening for Ovarian Cancer: A Reality Check
}

\author{
Stephanie Ricci • Ie-Ming Shih
}

Published online: 2 April 2013

(C) Springer Science+Business Media New York 2013

\begin{abstract}
Detection of stage I neoplastic disease has become the major goal for cancer prevention and reduction of cancer-associated mortality. Whereas this goal is realized in many human solid tumors, including carcinomas arising from breast, endometrium, prostate, and gastrointestinal tract, it has become a tantalizing objective in ovarian cancer. Several population-based clinical studies designed to screen for early stage ovarian cancer fail to provide a clinically satisfied positive predictive value and, as a result, lead to several unnecessary surgeries that are associated with higher morbidity and mortality. One of the reasons is that highgrade serous carcinoma - the most common and lethal ovarian cancer-may likely arise from fallopian tube epithelium and involve the ovary secondarily. Therefore, "ovarian" cancer is unlikely ever stage I at presentation. This commentary will briefly summarize the recent findings in ovarian cancer screening and discuss the challenges, promise, and reality for early detection of ovarian cancer.
\end{abstract}

Keywords Cancer prevention · Ovarian cancer · Early detection $\cdot$ Cancer screening - Ovarian cancer screening . Screening tools $\cdot$ Stage I neoplastic disease $\cdot$ Obstetrics and gynecology $\cdot$ Carcinoma $\cdot$ Prostate, lung, colorectal, and ovarian cancer $\cdot$ Screening $\cdot$ Serous tubal intraepithelial carcinoma

Ovarian cancer (high-grade serous carcinoma) is a disease with a low prevalence and a high mortality rate. Its lethality is mostly associated with diagnosis at an advanced stage (5-

\footnotetext{
S. Ricci $(\bowtie) \cdot$ I.-M. Shih $(\square)$

Departments of Gynecology and Obstetrics and Pathology, Johns Hopkins Medical institutions, 1550 Orleans Street, CRB2-305,

Baltimore, MD, USA

e-mail: sricci1@jhmi.edu

e-mail: ishih@jhmi.edu
}

year survival for stage III/IV disease are $27 \%$ and $16 \%$ respectively). Studies have demonstrated an improvement in survival with aggressive cytoreduction by a gynecologic oncologist and the introduction of platinum and taxanebased chemotherapy [1]. Despite these advances, 22,300 women are diagnosed with ovarian cancer each year in the United States and 14,300 women die of their disease [2]. Ovarian cancer is largely asymptomatic at an early stage and even at advanced stages symptoms often are vague, nonspecific, and difficult to discern. However, women who are diagnosed with ovarian cancer at an earlier stage have a much improved survival and it is therefore reasonable to devise a screening tool for this purpose. Early detection has proven difficult because definitive diagnosis is achieved only through an invasive surgical procedure. A screening test for ovarian cancer must therefore achieve a difficult balance to deliver sensitivity high enough to identify a disease with low prevalence and specificity high enough to spare women from unnecessary surgery. To date, bimanual pelvic examination has largely proven ineffective for early detection of ovarian cancer [3], resulting in a shift to the use of transvaginal ultrasound (TVU) and serum CA-125 levels. Unfortunately, neither method heralds a promising future, either individually or in combination. There are three ongoing, large, prospective trials employing TVU and CA-125 levels in the United States, United Kingdom, and Japan. None of these trials have yet reached an endpoint to analyze survival outcomes; however, preliminary results have been published.

In the United States, the Prostate, Lung, Colorectal and Ovarian (PLCO) cancer screening trial randomized 78,237 women between the ages of 55-74 to receive screening with CA-125 and TVU or no screening [4]. Of the women randomized to receive screening, 28,816 received at least one test. The results of the baseline screening examination revealed 1,338 (4.7\%) abnormal TVU examinations and 402 (1.4 \%) abnormal CA-125 serum levels. There were 29 
malignant neoplasms identified; 9 were tumors of lowmalignant potential, 1 was a malignant granulosa cell tumor, and 19 were invasive epithelial tumors. Of the epithelial tumors diagnosed, 15 (78.9 \%) of 19 patients were advanced stage (III/IV). The positive predictive value (PPV) for an abnormal CA-125 was $3.7 \%, 1.0 \%$ for an abnormal TVU, respectively, and $23.5 \%$ if both tests were abnormal. Importantly, $541(94.9 \%)$ of 570 women who underwent a surgical procedure for a positive screen did not have cancer. Recently, data from the first four annual screens were published [5]. The PPV remained similar for subsequent screening rounds; however, the number of surgeries performed for screen positive women decreased from $34 \%$ to $15-20 \%$. The overall ratio of surgeries to screen detected cancers remained high at 19.5:1 and $72 \%$ of screen detected cancers were advanced stage.

In Japan, 82,487 asymptomatic postmenopausal women were randomized to an intervention group or a control group and followed for an average of 9.2 years [6]. Women in the intervention group received sequential TVU and serum CA125 tests. Twenty-seven cancers were detected during the first screening round plus recall. Of the cancers identified, 11 (40. $7 \%$ ) of 27 cancer cases were grade 1 . Interestingly 17 (63\%) of 27 patients were stage 1 , whereas $9(33 \%)$ of 27 cases were advanced stage. Compared with the control group, 12 (38 \%) of 32 cancer cases were stage 1 and 18 (56\%) of 32 patients were advanced stage, conferring an increase in early-stage cancers detected in the screen group. Unfortunately, this difference was not statistically significant $(p=0.23)$. Furthermore, there were eight cancers that failed to be diagnosed by screening. PPV was not reported for TVU and serum CA-125; however, a total of 278 (91\%) of 305 women underwent surgery and did not have cancer. This amounted to an overall ratio of surgeries to screen detected cancer of 33:1.

The study in the United Kingdom has taken a slightly different approach. The trial arms were designed based on a prospective study also conducted in the United Kingdom by Menon et al. [7] which was based on a smaller prospective study that demonstrated a survival benefit for sequential screening with CA-125 followed by TVU [8]. In Menon's study, the CA-125 result for each postmenopausal woman was interpreted using a risk of ovarian cancer algorithm (ROC) and divided into low risk, intermediate risk, and elevated risk. Low-risk women continued with annual screening, whereas women in the intermediate risk group had repeated CA-125 testing and women with elevated risk underwent TVU. There were a total of 13,582 women randomized to screen or no screen. For primary invasive EOC, the specificity was $99.8 \%$ (95\% confidence interval (CI) 99 . 7-99.9) and PPV was $19 \%$ (95 \% CI 4.1-45.6). Of note, 16 women underwent surgery and 11 had benign diseases.

The UK Collaborative Trial of Ovarian Cancer Screening (UKCTOCS) includes a total of 202,638 postmenopausal women between the ages of 50-74 years randomized to one of three arms: no treatment, annual CA-125 screening with TVU follow-up (multimodal screening, MMS), or annual screening with TVU alone [9]. In the MMS group, there were 42 ovarian/tubal cancers detected, 8 of which were borderline tumors. There were 45 cancers detected in the TVU arm, 20 of which were borderline tumors. Of the total 58 invasive cancers in both groups, 28 (48\%) were stage I/II with no difference in detection rates between screening modalities. Interestingly, $63(65 \%)$ of 97 women in the MMS cohort and 820 (97\%) of 845 women in the TVU cohort underwent unnecessary surgery. Inclusive only of invasive epithelial ovarian/tubal cancers, the authors report sensitivity, specificity, and PPV of $89.5 \%, 99.8 \%$, and $35.1 \%$, respectively, for the MMS arm compared with $75 \%, 98.2 \%$, and $2.8 \%$, respectively, for the TVU arm. The specificity but not the sensitivity was statistically significant.

Although none of these trials have matured to the point of survival assessment, the preliminary results are not encouraging. The vast majority of cases identified by screening with TVU and serum CA-125 are borderline tumors or nonepithelial tumors, which have a good prognosis even at an advanced stage. The invasive epithelial cancers, which have the poorest prognosis and are presumably the target of

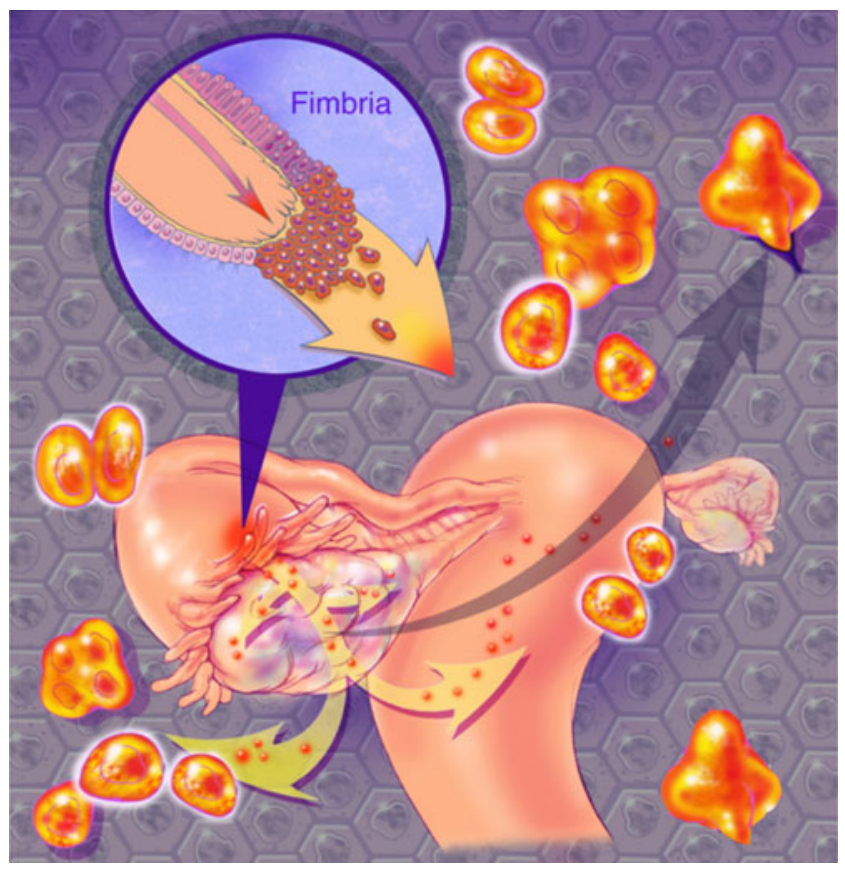

Fig. 1 Hypothesis for the tubal origin of ovarian high-grade serous carcinoma. During ovulation the dominant follicle ruptures, exposing fimbrial epithelium to a milieu of inflammatory factors and reactive oxygen species. This genotoxic environment supports the selection of TP53 mutations in epithelial cells, which clonally expand. Malignant transformation may occur and manifest as serous tubal intraepithelial carcinoma (STIC). These malignant cells can now shed onto ovarian and peritoneal surfaces to become "ovarian" serous carcinoma. (C John Hopkins University) 
generalized screening, fail to be recognized at an early stage by screening. In the Japanese study, eight women undergoing screening were found to have ovarian cancer after completion of the trial. These cases were predominantly serous epithelial ovarian cancer at an advanced stage, and they were presumably missed by screening at an early stage. Furthermore, the high number of women subjected to unnecessary surgery reported in all of these trials is unacceptable.

What can be learned from these findings is that a serum marker may be more precise than an imaging modality; however, the lack of specificity inherent to serum CA-125 levels does not recommend its candidacy as an effective screening biomarker. Part of the problem with the current screening tools may lie with our understanding of ovarian carcinogenesis. Up until now, "ovarian" cancer (high-grade serous carcinoma) has been thought to originate in the ovary and, appropriately, screening tools developed have thus focused. There is now new evidence to suggest that invasive epithelial cancer actually originates in the fallopian tube by the identification of a precursor lesion called serous tubal intraepithelial carcinoma or STIC (Fig. 1) [10, 11]. In light of this hypothesis, screening tools focused on the ovary are destined to miss early-stage disease because once the ovary is involved, cancer has already disseminated within the abdominal cavity. If the tubal origin of ovarian (high-grade serous) carcinoma is valid, the goal of detecting stage I ovarian cancer through screening is implausible. Instead future efforts should be invested in detecting low-volume ovarian cancer at a stage that promotes optimal debulking surgery, because mounting evidence has demonstrated that reduced residual tumor volume after cytoreduction surgery is positively correlated with better clinical outcome [1, 12, 13]. Because cells and/or DNA from high-grade serous carcinomas that originate from the fallopian tube may be transported into the uterine cavity and cervix, it is possible that a relatively noninvasive method can be designed to detect mutated DNA released from serous carcinoma cells. To this end, the research group from Vogelstein and Diaz has recently devised a novel approach to detect mutated genes from DNA obtained from liquid-based Pap smear specimens obtained during routine pelvic examination [14]. The investigators were able to detect the same mutations in DNA from liquid Pap smear specimens in $41 \%$ of ovarian cancers and in $100 \%$ of endometrial cancers. Moreover, based on a small set of 12 genes and a sequence-based method that can be feasibly adapted to a molecular diagnostic laboratory, the research team could detect cancer without previous knowledge of the tumor's genotype to query mutations. Given these promising results, future studies will focus on whether molecular tests can diagnose asymptomatic ovarian cancer patients with low-volume disease and determine if introducing new molecular tests, such as a standard liquid-based Pap smear, results in better clinical outcome.

\section{References}

1. Bristow RE, Tomacruz RS, Armstrong DK, Trimble EL, Montz FJ. Survival effect of maximal cytoreductive surgery for advanced ovarian carcinoma during the platinum era: a meta-analysis. J Clin Oncol. 2002;20:1248-59.

2. Cancer Facts and Figures 2012. http://www.cancer.org/research/ cancerfactsfigures/cancerfactsfigures/cancer-facts-figures-2012

3. Smith HL, Oi RH. Detection of malignant ovarian neoplasms: a review of the literature. I. Detection of the patient at risk; clinical, radiological and cytological detection. Obstet Gynecol Surv. 1984:39:313-28.

4. Buys SS, Partridge E, Greene MH, Prorok PC, Reding D, Riley TL, et al. Ovarian cancer screening in the Prostate, Lung, Colorectal and Ovarian (PLCO) cancer screening trial: findings from the intial screen of a randomized trial. AJOG. 2005;193:1630-9.

5. Partridge E, Greenlee RT, Xu JL, Kreimer AR, Williams C, Riley $\mathrm{T}$, et al. Results from four rounds of ovarian cancer screening in a randomized trial. Obstet Gynecol. 2009;113(4):775-82.

6. Kobayashi H, Yamada Y, Sado T, Sakata M, Yoshida S, Kawaguchi R, et al. A randomized study of screening for ovarian cancer: a multicenter study in Japan. Int J Gynecol Cancer. 2008;18:414-20.

7. Menon U, Skates SJ, Lewis S, Rosenthal AN, Barnaby R, Sibley $\mathrm{K}$, et al. Prospective study using the risk of ovarian cancer algorithm to screen for ovarian cancer. J Clin Oncol. 2005;23:7919 26.

8. Jacobs IJ, Skates SJ, MacDonald N, et al. Screening for ovarian cancer: a pilot randomized controlled trial. Lancet. 1999;353:120710.

9. Menon U, Gentry-Maharaj A, Hallett R, Ryan A, Burnell M, et al. Sensitivity and specificity of multimodal and ultrasound screening for ovarian cancer, and stage distribution of detected cancers: results of the prevalence screen of the UK Collaborative Trial of Ovarian Cancer Screening (UKCTOCS). Lancet Oncol. 2009;10:32740.

10. Piek JM, van Diest PJ, Zweemer RP, et al. Dysplastic changes in prophylactically removed Fallopian tubes of women predisposed to developing ovarian cancer. J Pathol. 2001;195:451-6.

11. Kuhn E, Kurman RJ, Shih IM. Ovarian cancer is an imported disease: fact or fiction? Curr Obstet Gynecol Rep. 2012;1(1):1-9.

12. Chang SJ, Bristow RE. Evolution of surgical treatment paradigms for advanced-stage ovarian cancer: redefining 'optimal' residual disease. Gynecol Oncol. 2012;125(2):483-92.

13. Chi DS, Eisenhauer EL, Zivanovic O, Sonoda Y, Abu-Rustum NR, Levine DA, et al. Improved progression-free and overall survival in advanced ovarian cancer as a result of a change in surgical paradigm. Gynecol Oncol. 2009;114(1):26-31.

14. Kinde I, Bettegowda C, Wang Y, Wu J, Agrawal N, Shih Ie M, et al. Evaluation of DNA from the Papanicolaou test to detect ovarian and endometrial cancer. Sci Transl Med. 2013;5(167):167. 